\title{
Strong effect of precursor preparation on the morphology of semicrystalline phase inversion poly(vinylidene fluoride) membranes
}

\author{
Dar-Jong Lin ${ }^{a}$, Konstantinos Beltsios ${ }^{\mathrm{b}}$, Tai-Horng Young ${ }^{\mathrm{c}}$, Yi-Su Jeng ${ }^{\mathrm{a}}$, Liao-Ping Cheng ${ }^{\mathrm{a}, *}$ \\ a Department of Chemical and Materials Engineering, Tamkang University, Taipei 25137, Taiwan \\ ${ }^{\mathrm{b}}$ Department of Materials Science and Engineering, University of Ioannina, Ioannina, GR-45110, Greece \\ c Institute of Biomedical Engineering, College of Medicine and College of Engineering, National Taiwan University, Taipei 10016, Taiwan
}

Received 19 December 2004; received in revised form 22 July 2005; accepted 28 July 2005

Available online 9 September 2005

\begin{abstract}
This work documents the role of precursor preparation as an overlooked important factor in the control of membrane structures obtained by the phase inversion (PI) of solutions of crystallizable polymers. The effect of precursor-solution preparation temperature on the structure of a phase inversion poly(vinylidene fluoride) (PVDF) membrane is examined. The phase inversion example explored is the coagulation of a PVDF solution in dimethyl formamide (DMF) by the action of 1-octanol, which is a soft non-solvent. Polymer dissolution temperature is varied from 50 to $110^{\circ} \mathrm{C}$ and the immediate consequence is a substantial variation in the density of nuclei available for the initiation of crystallization. Membrane structure is defined through a competition between solid-liquid (s-1) and liquid-liquid (1-1) equilibria; such competition can be strongly affected by changes in the density of crystallization nuclei. As a result, it is found possible to generate a wide spectrum of membrane morphologies without resorting to a variation of any of the standard parameters, such as dope and coagulant compositions and temperatures, usually employed for the control of phase inversion membrane morphology. It is also found that aging time can be used as a further structure-control parameter and a 'maturation time-dissolution temperature' equivalence is demonstrated.
\end{abstract}

(C) 2005 Elsevier B.V. All rights reserved.

Keywords: Precursor preparation; Poly(vinylidene fluoride); Membranes; Phase inversion; Phase separation

\section{Introduction}

Phase inversion (PI) is the leading method for the preparation of polymeric membranes with a porous bulk part [1-3]. PI involves the coagulation of a concentrated polymer solution precursor known as the 'dope' by a change in composition. The change in composition can be achieved either by the introduction of new species or through the selective removal of species. The introduction of new species is usually accomplished by immersion of the dope in a bath that is solvent-miscible but a non-solvent of the polymer. PI through the removal of dope species involves dopes containing a volatile solvent and a non-volatile non-solvent; gradual evaporation leads to the preferential removal of solvent species.

\footnotetext{
* Corresponding author. Tel.: +886 2 6215656; fax: +8862 6252770 .

E-mail address: lpcheng@ mail.tku.edu.tw (L.-P. Cheng).
}

In both cases the change in composition brings the solution within a multiphase region of the phase diagram. PI membranes can be: (a) symmetric (morphology is fixed along the thickness) or (b) asymmetric (morphology varies substantially along the thickness). While early PI research focused almost exclusively on polymers with limited or no capacity for crystallization, in recent years the additional structural options offered by the processing of dopes of crystallizable polymers have led to the establishment of widely pursued new directions in PI research [4-7]. The latter research is in many respects parallel to a membrane fabrication method known as thermally induced phase separation (TIPS), as the latter is usually applied to semicrystalline polymers and also employs a change in the quality of a solvent (through a change of temperature, instead of a change in composition, as in PI) [8-10].

For both PI and TIPS of crystallizable polymers, a competition of solid-liquid (s-l) equilibria and liquid-liquid (l-l) 
equilibria is possible, though frequently only one of the two types of equilibria plays a major role in the definition of final morphology [4-10]. Recently, we employed a poly(vinylidene fluoride) (PVDF)/dimethyl formamide (DMF) dope coagulated in 1-octanol in order to explore a PI case where both types of equilibria have a substantial effect on membrane morphology [11].

In the case of PI, it is well established that the membrane morphology can change dramatically by a substantial change in the composition and/or temperature of the dope, the coagulant or both [1-15]. On the other hand, the exact route of preparation of the PI dope is usually overlooked as a parameter capable of affecting membrane morphology; pertinent concerns are usually limited to the filtering of the dope to avoid defective structures as a result of the presence of sizable inclusions. A PI system for which both liquid-liquid and liquid-solid separations can play an important role in final morphology is appropriate for the exploration of the potential effect of subtle variations in the method of dope preparation; the aforementioned PVDF/DMF/1-octanol PI case offers a convenient test system.

Dopes of a given composition are prepared by dissolution of PVDF in DMF in the widest, practically, possible range of temperatures $\left(50-110^{\circ} \mathrm{C}\right)$, then cooled to room temperature and finally coagulated in a 1-octanol bath. It is reported herein that a variation of dope preparation temperature alone can lead to an impressive variation in membrane structure. For instance, the size of the semicrystalline particles in the formed membrane increased from ca. 0.5 to $15 \mu \mathrm{m}$, as the dope preparation temperature was raised from 50 to $110^{\circ} \mathrm{C}$. Such a dramatic change in particle size (or equivalently nucleation density) was attributed to the difference in the state of dissolution of the metastable dopes. The latter was evident from the maturation study of the dopes by X-ray diffraction method; viz., the diffraction intensity increased as the maturation time increased until the dopes eventually gelled.

\section{Experimental}

\subsection{Materials}

PVDF (Kynar 740 from Elf Atochem; intrinsic viscosity $=0.881 \mathrm{dL} / \mathrm{g}, \mathrm{Mn}=254,000 \mathrm{~g} / \mathrm{mole}$ ) was obtained in pel- let form. DMF (Baker Analyzed, reagent grade, 98 wt.\%, $d=0.944 \mathrm{~g} / \mathrm{ml}$ ) was used as the solvent. 1-Octanol (Reidel-de Haen, reagent grade, $d=0.82 \mathrm{~g} / \mathrm{ml}$ ) was used as the nonsolvent. All materials were used as received.

\subsection{Membrane preparation and characterization}

PVDF membranes were prepared by the phase inversion method [11]. In brief, PVDF was dissolved at 50, 60, 70, 90 or $110^{\circ} \mathrm{C}$ in DMF in a sealed glass bottle to form a $23 \mathrm{wt} . \%$ homogeneous dope solution. The time for dissolution varied with temperature and it was, in all cases, slightly longer that necessary for a clear solution to form. Typical dissolution times appear in Table 1. Following dissolution, all solutions were cooled to $25^{\circ} \mathrm{C}$, stood for $1 \mathrm{~h}$, cast on a glass plate, and then immersed immediately into a 1-octanol $25^{\circ} \mathrm{C}$ bath to induce polymer precipitation. The nascent membrane was washed in a series of non-solvents to extract residual DMF and 1-octanol. Subsequently, the membrane was press-dried between sheets of filter papers at ca. $45^{\circ} \mathrm{C}$.

The nano-scale morphologies of the top and bottom surfaces and the bulk of the membranes were observed using a low voltage field emission scanning electron microscope (FESEM, Carl Zeiss, Leo 1530, Germany). The sample was vacuum-dried and attached to a sample holder using conductive copper tapes. Silver paste was applied at the edges of the sample in order to enhance electronic conductivity. Subsequently, the sample was sputtered with a thin layer (ca. $1-2.5 \mathrm{~nm}$ ) of Pt-Pd alloy and observed under $1-3 \mathrm{kV}$ acceleration voltages with an in-lens detector. The thermal behavior of the PVDF membranes was examined using DSC (Netzsch, DSC 200, Germany). The instrument was calibrated with an indium standard before running the tests. An appropriate amount of membrane sample (typically $5 \mathrm{mg}$ ) was sealed in an aluminum pan and placed in the heating chamber together with an empty pan used as a reference. The temperature was raised from 25 to $250{ }^{\circ} \mathrm{C}$ at a heating rate of $10^{\circ} \mathrm{C} \mathrm{min}-1$ under nitrogen flow. The melting temperature and the heat of fusion were determined from the obtained thermogram. Wide angle X-ray diffraction (Bruker, D8 Advance, Germany) analyses of the membranes were performed to disclose the crystal structures of the membranes. The wavelength of the X-ray was $1.54 \AA$ (copper $\mathrm{K} \alpha$ line), the width of the slit was $0.6 \mathrm{~mm}$, and the scanning rate was $0.12^{\circ} \mathrm{min}^{-1}$ with a

Table 1

The conditions of the dopes and the strengths of the membranes

\begin{tabular}{llccc}
\hline Dope condition & & & \\
\hline Membrane code & $\begin{array}{l}\text { Dissolution } \\
\text { temperature }\left({ }^{\circ} \mathrm{C}\right)\end{array}$ & Dissolution time (h) & Gelation time (d) & $\begin{array}{l}\text { Tensile strength/elongation } \\
\left(\mathrm{g}_{\mathrm{f}} \mathrm{mm}^{-2} / \mathrm{mm}\right)\end{array}$ \\
\hline M50 & 50 & 68 & 7 & $135 / 2.3$ \\
M70 & 70 & 9 & 12 & $110 / 1.6$ \\
M90 & 90 & 5 & 82 & $82 / 1$ \\
M110 & 110 & 4 & $>130$ & $73 / 0.92$ \\
\hline
\end{tabular}

Coagulation bath: 1-octanol at $25^{\circ} \mathrm{C}$. Gelation time was defined as the period of time for a dope solution to gel. A gel sample is one which does not flow when the sample bottle is turned upside-down. 
resolution of $0.01^{\circ}$. Tensile strengths at break (Dachang, QC512, Taiwan) of the membranes were determined following ASTM (D638 IV). Polarized optical microscopy (POM) was also applied for the study of membrane samples, but only to a limited extent, as a result of the small scale of most structural features and the problem of substantial opacity. For the reduction of opacity the membranes were soaked in ethanol (a non-solvent for PVDF) during imaging.

\subsection{Maturation of the dopes and membrane preparation}

Dopes prepared at different dissolution temperatures and cooled to $25^{\circ} \mathrm{C}$ were aged at the latter temperature for an extended period of time. At different times during storage, samples were analyzed by X-ray diffraction. A suitable amount of the sample was poured into a liquid holder for the XRD and then it was X-ray scanned at a relatively fast rate $\left(2 \theta=3^{\circ} \mathrm{min}^{-1}\right)$ in order to minimize the possibility of gelation at the sample surface caused by humidity. In order to examine the effect of dope maturation on the membrane morphology, membranes were prepared using dopes with different aging times by the immersion-precipitation method as described above. The porous structures of the formed membranes were observed by FESEM.

\section{Results and discussion}

\subsection{Morphology of the membranes}

The effect of dope dissolution temperature on the morphology of the membrane is demonstrated in Figs. 1-4. For the case of immersing a dope dissolved at $50^{\circ} \mathrm{C}$ in 1-octanol, as shown in Fig. 1a, the cross section of the membrane (M50, Table 1) is composed of small interconnected particulate entities with a globule-like shape, ca. 0.2-0.6 $\mu \mathrm{m}$ in diameter. The network of globular particles coexists with a pore network. As both the top and the bottom surfaces are porous and also because porous structure does not vary along the thickness of the sample, this membrane can be considered to be of the "symmetric" type, despite some difference in the appearance of the two surfaces. At a high magnification $(100 \mathrm{k} \times)$, Fig. $1 \mathrm{~b}$ shows the fine structure of several globules. Their surfaces are very rough with a granular fine structure having a characteristic dimension of ca. $20 \mathrm{~nm}$. Also observed are fibril, stick, sheaf, and sheet-like elements contributing to the connectivity of the globular elements. The thinnest identifiable width of the connecting elements is ca. $10 \mathrm{~nm}$, corresponding to the thickness of a PVDF lamella, as reported in the literature [16-18]. The formation mechanism of the spherulitic globules has been analyzed in a recent publication [11]. In brief, there are three main stages involved: (i) precipitation initiates by nucleation and growth of PVDF spherulites and structurally related entities with a total extent of crystallization insufficient for liquid-liquid separation. (ii) Upon substantial increase of the total extent of polymer crystalliza-

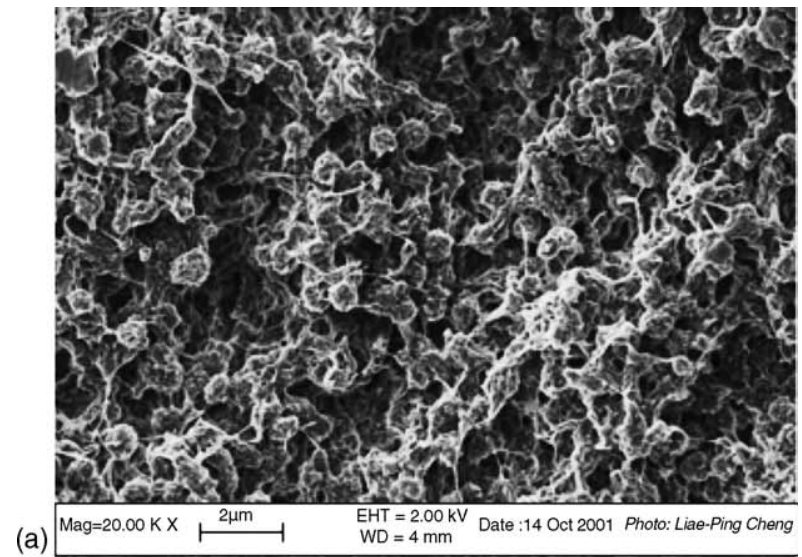

(a)

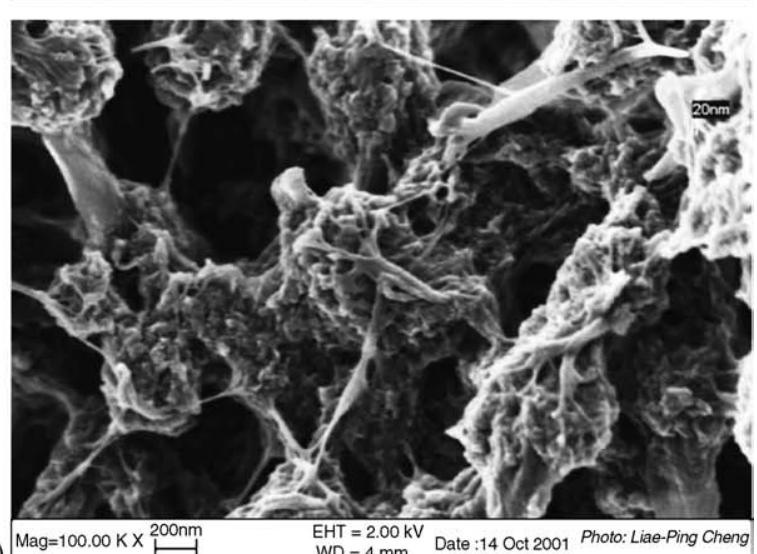

(b)

Mag= $100.00 \mathrm{~K} \times \stackrel{200 \mathrm{~nm}}{\longmapsto}$

WD $=4 \mathrm{~mm}$ Date $: 14$ Oct 2001 Photo: Liae-Ping Cheng

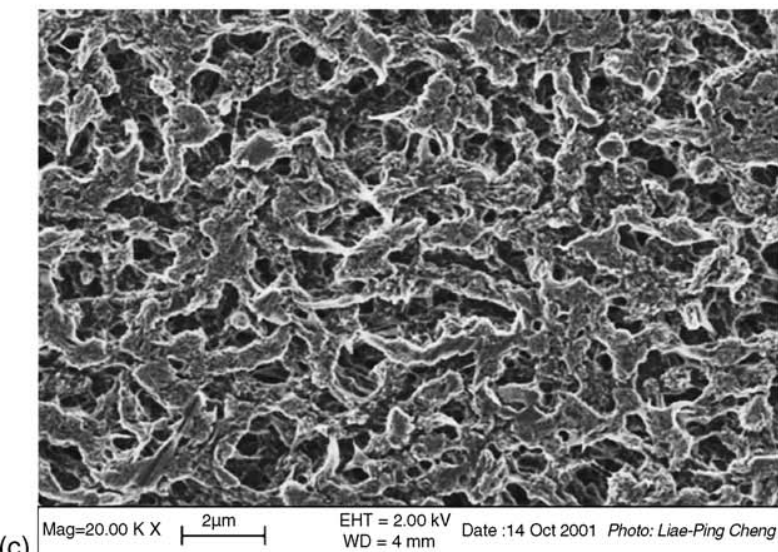

(c) $\mathrm{EHT}=2.00 \mathrm{kV}$
$\mathrm{WD}=4 \mathrm{~mm}$

Fig. 1. SEM micrographs of the PVDF membrane prepared by immersion of a $50{ }^{\circ} \mathrm{C}$ dissolved dope in 1-octanol. (a) cross section; (b) high magnification of (a); (c) bottom surface.

tion, liquid-liquid separation becomes possible and generates polymer-rich and polymer-poor liquid domains. (iii) Final stage precipitation leads to nanograined shells at the globular surface. The bottom surface, as shown in Fig. 1c, is porous as the cross section. The particles here are flattened (i.e., truncated globules) as a result of their growth against the glass substrate, and they join with each other to form elongated patterns.

Fig. 2 shows the morphology of the membrane (M70, Table 1) prepared by immersion of a dope dissolved at $70^{\circ} \mathrm{C}$ 


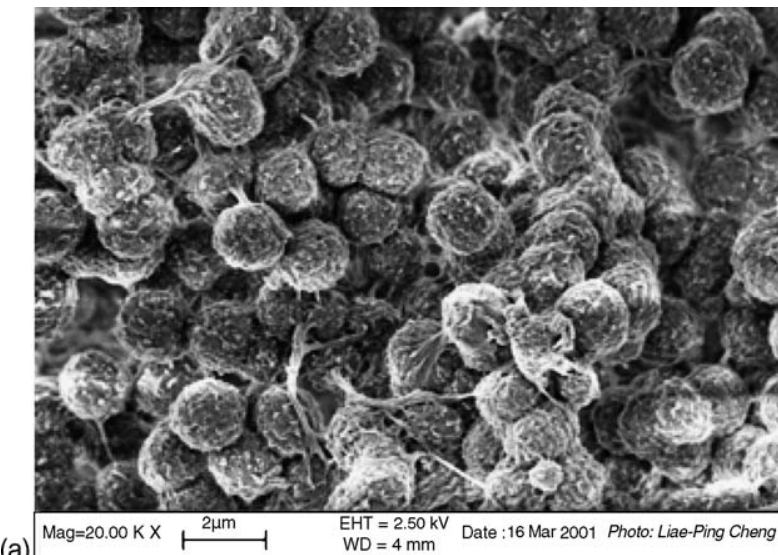

(a)

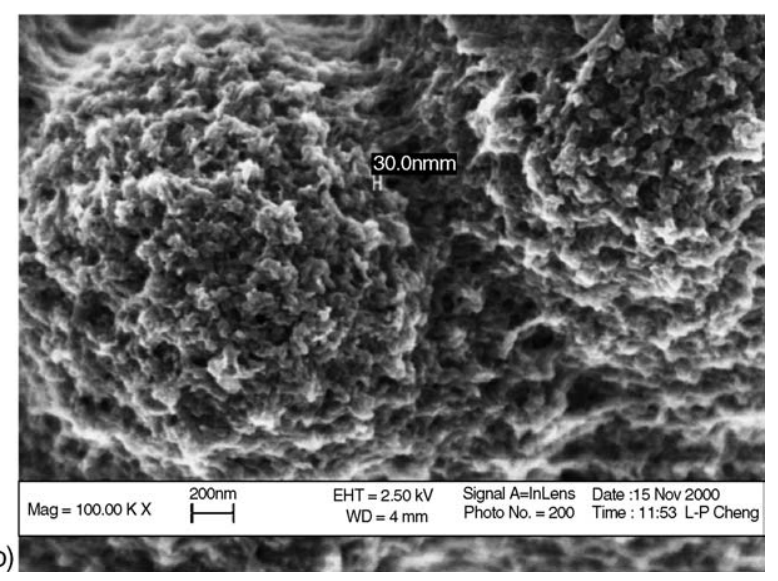

Fig. 2. SEM micrographs of the cross section of the PVDF membrane by immersion of a $70^{\circ} \mathrm{C}$ dissolved dope in 1-octanol. (a) low magnification; (b) high magnification.

in 1-octanol. Compared with Fig. 1, the largest structural entities (globules) are more round and uniform in size, which is in the range of 1.5-2.0 $\mu \mathrm{m}$ i.e. a few times (3-5) larger than that of Fig. 1 globules. The surfaces of the globules, as shown in Fig. 2b, are porous and also covered with nanograins. Various nano-scale morphological features of this membrane, such as twisted lamellae, branching sheaf, truncated globules, etc. have been identified and discussed previously [11]. The membrane prepared by using a $90{ }^{\circ} \mathrm{C}$-dissolved dope is shown in Fig. 3 (M90, Table 1). Compared to M70 samples, the globules are further perfected and larger in diameter, though, overall, these two membranes exhibit similar morphological features. A high magnification image of a typical globule appears in Fig. 3b. Quite interestingly, a primitive crystallite in the shape of twisted sheaf is found to seed on the granular surface of the globule. This crystal element would have grown into a side-on globule, if its growth had not been terminated during the precipitation process. Compared with Fig. 1b or Fig. 6 in [11], similar side-on crystals are also observable (a clear evidence of heterogeneous nucleation) even though there are large differences in the sizes of the spherulites in these membranes. The bottom surface of the membrane, as shown in Fig. 3c, represents a good example of a flattened
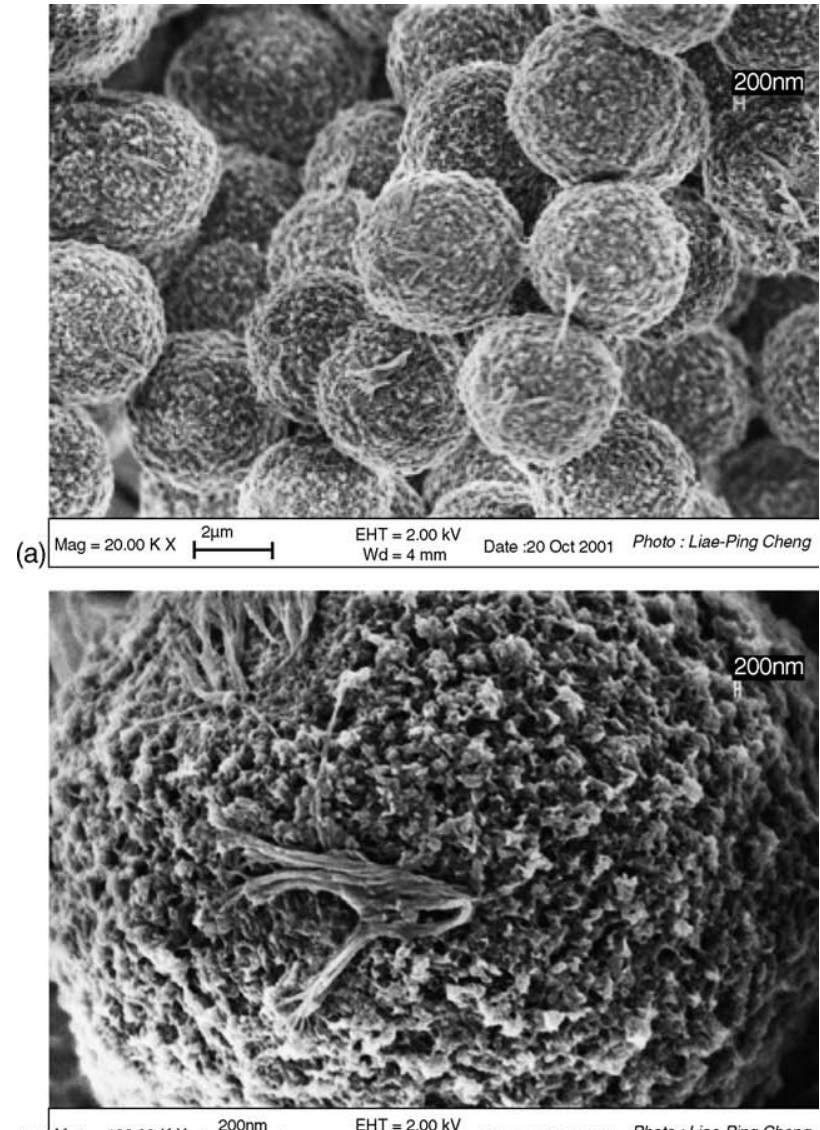

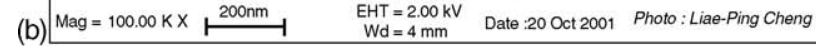

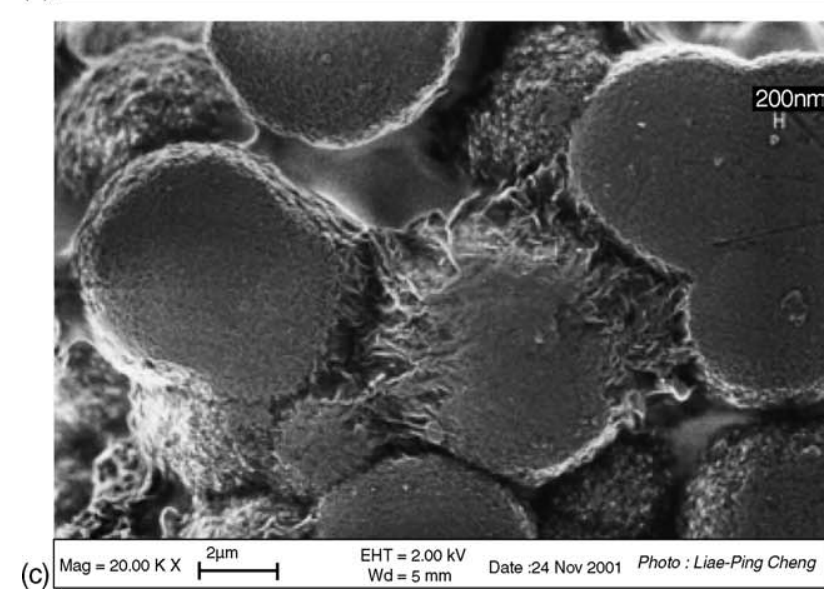

Fig. 3. SEM micrographs of the cross section and bottom surface of a PVDF membrane. Dope dissolution temperature $=90^{\circ} \mathrm{C}$. (a) cross section; (b) high magnification of (a); (c) bottom surface.

structure. Although the latter structure appears to be dense and featureless, further magnification (not shown) indicates a rough surface consisting of elongated nanograins of size $10-20 \mathrm{~nm}$.

When the dope dissolution temperature was further raised to $110^{\circ} \mathrm{C}$, a mechanically weak membrane was formed. The SEM image of this membrane is shown in Fig. 4. It is composed of very large globules that cover a wide range of sizes (ca. $15-50 \mu \mathrm{m}$ ). Comparison of Figs. 1 and 4a suggests that 
(a)
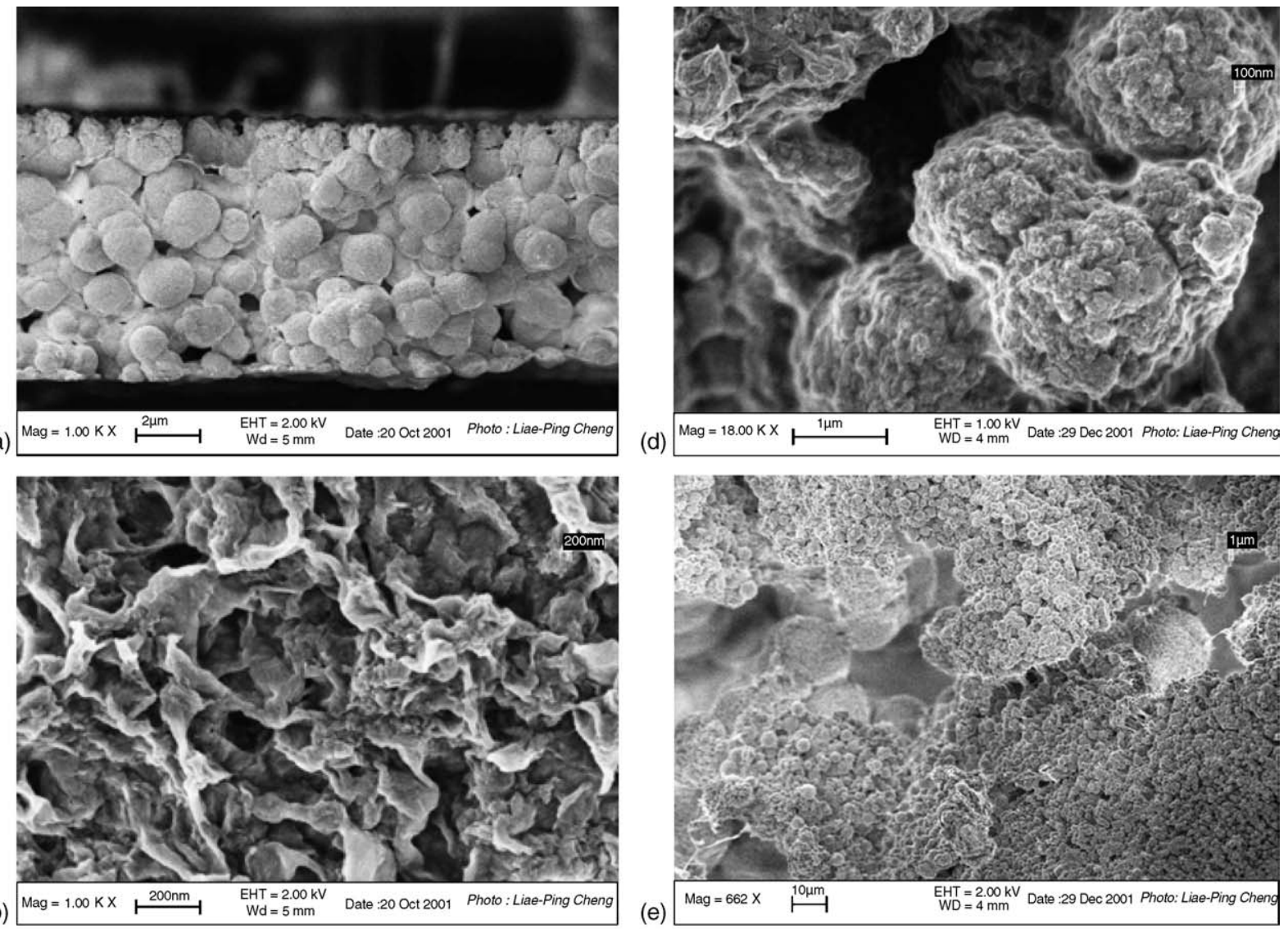

(d)
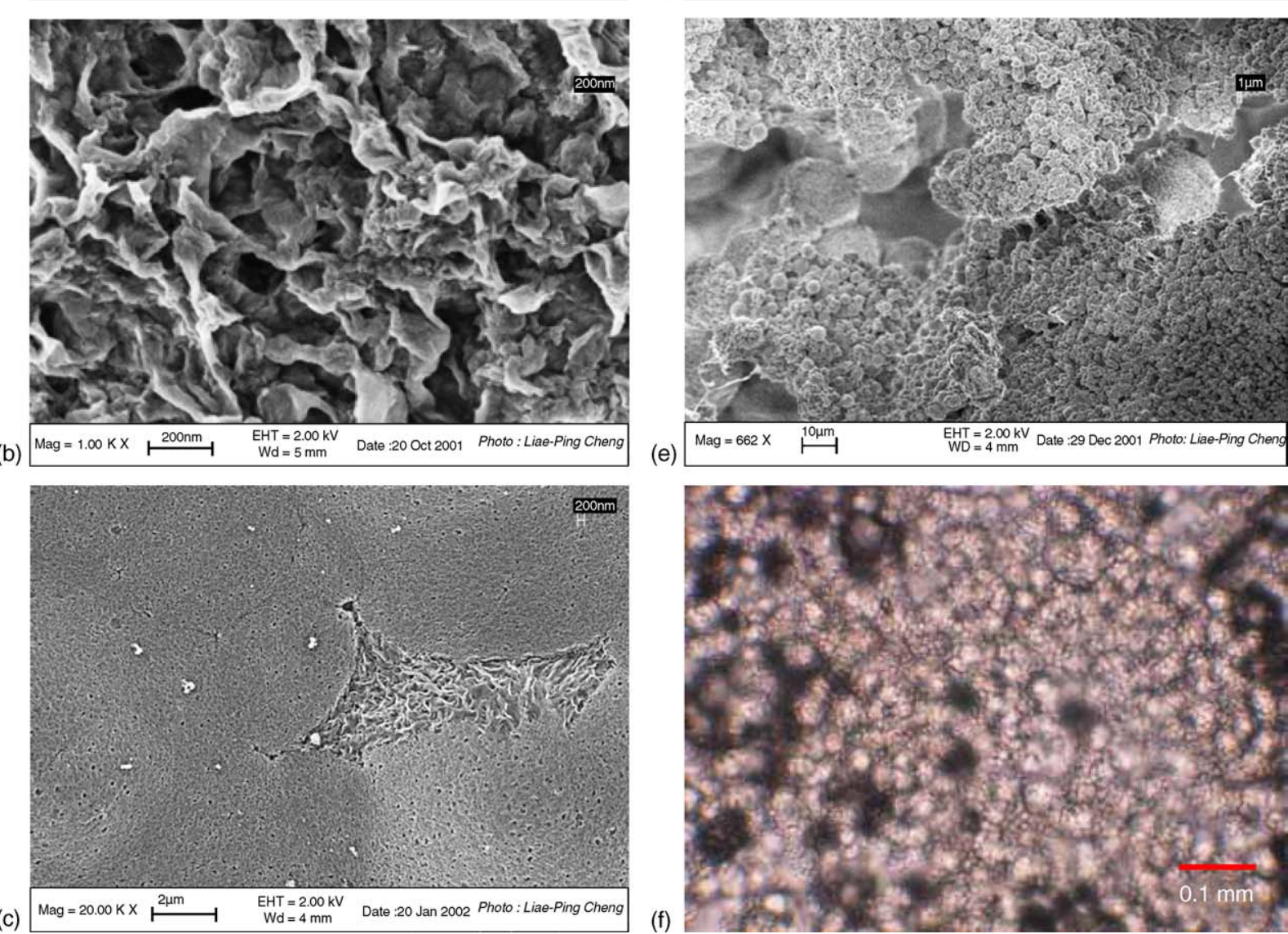

(e)

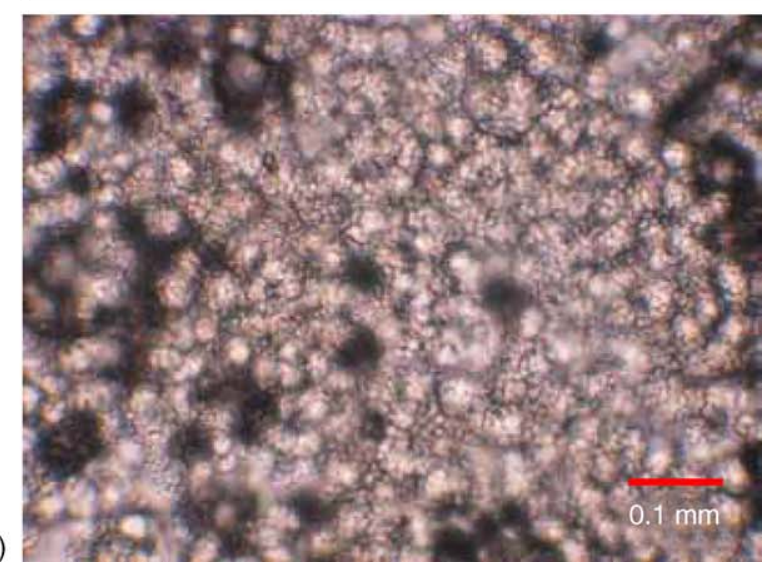

Fig. 4. SEM micrographs of the PVDF membrane by immersion of a $110^{\circ} \mathrm{C}$ dissolved dope in 1 -octanol. (a) cross section; (b) high magnification of (a); (c) top surface; (d) bottom surface; (e) bottom surface at reduced magnification; (f) cross-polarized POM micrograph of the bottom surface.

the linear scale $(\mathrm{d})$ of globular structure can change by full two orders of magnitude ( +100 times), through a careful tuning of the often neglected details of pre-coagulation preparation steps. Also, as there is primarily one nucleus per globule and the latter has a volume on the order of $\mathrm{d}^{3}$, it follows that the tuning of pre-coagulation conditions allows for a variation of the nuclei density by six orders of magnitude.

The integrity of the M110 membrane primarily stems from the presence of a skin-like tight layer at the top surface (Fig. 4c). In the absence of this layer, the loosely held together globules may fall apart upon lightly rubbing. For the preparation conditions in discussion, the mechanical strength of the membranes deteriorates with increasing dope preparation temperature (Table 1). The latter finding can be attributed to the fact that increasing preparation temperatures lead to globules having a density of interconnecting material that does not scale with the volume of globules. In terms of micro filtration operations, membranes M90 and M110 are too weak to be usable as prepared. The nano-structure of the surface of a large M110 globule is shown in Fig. 4b. Unlike the glob- 
ules shown previously in Figs. 2 and 3b, nanograins do not cover densely the surface, but are found inter-dispersed with twisted sheet-like crystals. The thickness of the crystal sheets is ca. $10-20 \mathrm{~nm}$.

The morphology of the bottom surface is shown in Fig. $4 \mathrm{~d}$. Its structure consists of smaller globules ca. $1 \mu \mathrm{m}$ in diameter. The structure of these globules appears to be intermediate of those of the globules found in the bulk of the M50 and the M70 membranes. Its occurrence must be attributed to heterogeneous nucleation at the surface of the glass plate, which supports the dope during coagulation. Given the rarity of primary nuclei in the M110 dope, heterogeneous nucleation will overtake structure formation at the glass plate.

An image of the bottom surface of the M110 membrane at a lower magnification (Fig. 4e) shows that some structural inhomogeneity and additional structural features are present at a larger scale. In the right side of the micrograph the globules of ca. $1 \mu \mathrm{m}$ in diameter are sufficiently distinct, despite some tendency for clustering in groups (a tendency also observable in Fig. 4b). In the left side of the same micrograph some individual globules are larger, while at the same time some dense and irregular clusters, ca. 5-10 $\mu \mathrm{m}$ in diameter, are seen. Some openings in the range of few tens of $\mu \mathrm{m}$ are also observed. Finally, a POM photograph of a similar (bottom) surface is shown in Fig. 4f. Here, the light color is indicative of the presence of crystallinity in the solid domains; membrane crystallinity is quantified in the next section by different means. As the probing method is different and the depth of field in the case of Fig. $4 \mathrm{f}$ is more limited than in the case of Fig. 4e, an additional structural feature, corresponding to clusters with a diameter in the range of $30 \mu \mathrm{m}$, appears to prevail. There is also a finer structure within the $30 \mu \mathrm{m}$ features of Fig. 4f; this finer structure belongs to the range of $5-10 \mu \mathrm{m}$ and can be correlated with the various clusters seen in Fig. 4e. Finally, the dark gaps in Fig. $4 \mathrm{f}$ correspond precisely to the large openings seen in Fig. 4e.

\subsection{DSC and X-ray analysis}

The thermal behavior and crystal structure of membranes prepared by dopes dissolved at different temperatures were examined by DSC and WAXD and the results are shown in Figs. 5 and 6, respectively. From Fig. 5, it can be seen that all membranes exhibit a melting peak around $170^{\circ} \mathrm{C}$ at the scanning rate of $10^{\circ} \mathrm{C} \mathrm{min}^{-1}$. The heats of fusion for these membranes are all measured to be ca. $52 \mathrm{~J} / \mathrm{g}$, corresponding to a crystallinity of $50 \%$ (a pure PVDF crystal has a $\left.\Delta H^{\circ}=105 \mathrm{~J} / \mathrm{g}[16,19]\right)$. Despite the significant morphological difference in these membranes, the corresponding thermograms exhibit only minute differences in the $60-110^{\circ} \mathrm{C}$ range; such differences are also reflected in WAXD patterns (see below).

The X-ray diffraction patterns of various membranes are shown in Fig. 6. On the basis of crystallographic data in the literature, it is concluded that all membranes are of $\alpha$-type PVDF crystal structure [20-22]. The strong diffraction peaks

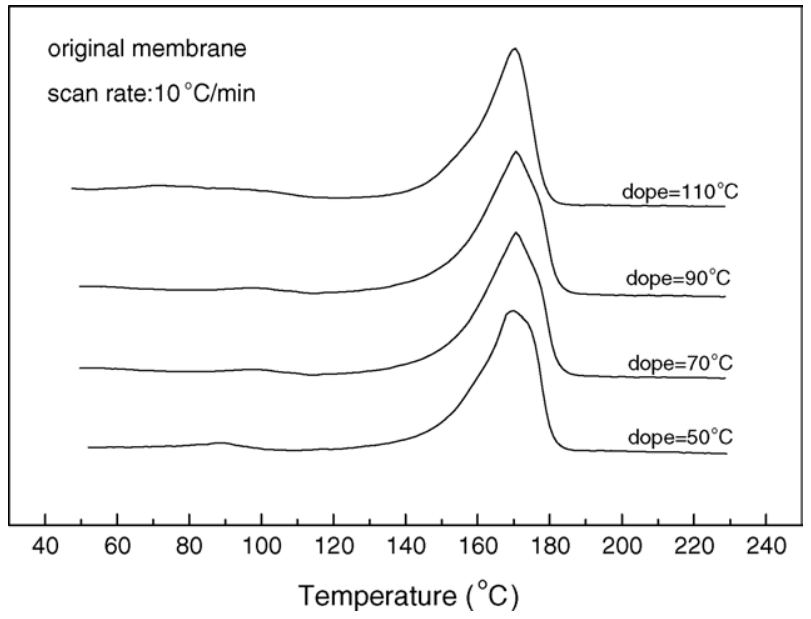

Fig. 5. DSC thermograms of PVDF membranes prepared by using dopes dissolved at different temperatures.

at $18.4,20.1$, and $26.6^{\circ}$ represent the reflections of $(200)$, (1 10$),(201)$ planes, respectively. Other peaks are relatively weak and their indices are shown in Fig. 6, which matches an orthorhombic unit cell with cell constants of 9.66, 4.96, and $4.64 \AA$. The membranes prepared by dopes dissolved at 50,70 , and $90^{\circ} \mathrm{C}$ exhibit similar diffraction intensities over the entire $2 \theta$ range. The only noticeable difference is at $2 \theta=18.4^{\circ}(200)$ at which angle only a shoulder is observed for membrane M90, whereas for membrane M50 there is a small peak. Membrane M70 is the intermediate of membranes M50 and M90. By contrast, membrane M110 shows a diffraction pattern with much stronger (2 00 ) and (201) diffractions than the other three membranes. It is interesting to note that although there is large difference in nucleation density among the four membranes (M50 > M70 > M90 > M110), the overall crystallinity of these membranes remains unaffected.

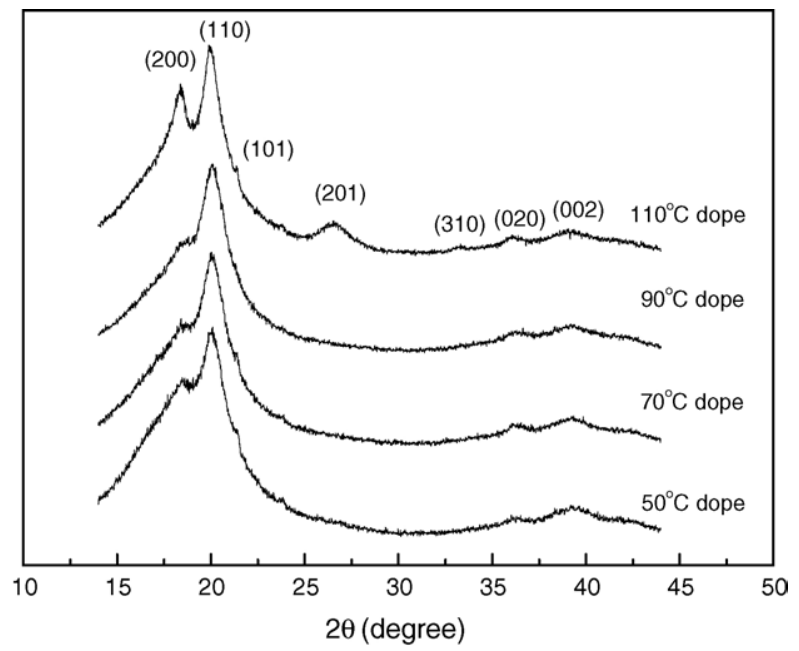

Fig. 6. WAXD patterns of PVDF membranes prepared by using dopes dissolved at different temperatures. 


\subsection{Effect of dope maturation}

Figs. 1-4 clearly indicate that the final morphology of the PVDF membrane was affected most significantly by the nucleation density; the higher the latter is, the smaller the size of the globule and the more compact and stronger the resulting membrane is. Figs. 1-4 also suggest that as the temperature for dope preparation was changed, the nucleation density in the membrane changed accordingly. In other words, casting dopes prepared at different temperatures may be at different levels of dissolution such that they contained different amount of pre-nucleation aggregates, which nucleated and grew upon contact with the non-solvent, 1-octanol. In previous investigations of aliphatic polyamide (Nylon-6, -66 , and -610) membrane formation, the state of aggregation of the casting dope was adjusted by addition of non-solvent or nucleation seeds in the dope $[5,23,24]$. In some extreme cases, metastable dopes on the edge of precipitation with respect to crystallization were prepared, which contained a large population of pre-nucleation aggregates. Upon immersing them into a soft bath, skinless uniform microporous membranes composed of interlinked rod-like crystallites could be obtained. In the present research, it is interesting to note that
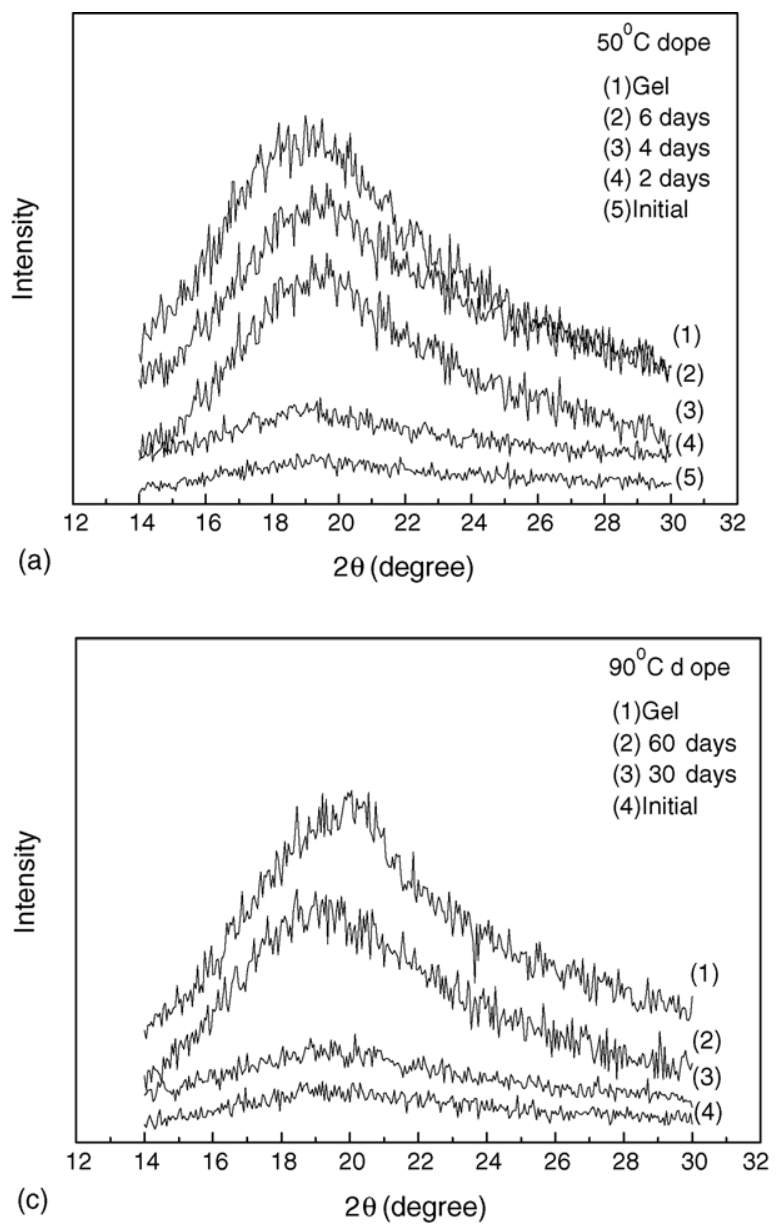

the state of the dope can be effectively controlled exclusively by the choice of dissolution temperature of PVDF in DMF. Table 1 shows the gelation times at $25^{\circ} \mathrm{C}$ for various dopes. For a dope dissolved at a higher temperature, the gelation time appears to be longer. For example, dope M50 took ca. 7 days to gel, while for dope M90 the corresponding time was 82 days. Finally, dope M110 remained in the state of a clear solution even after 130 days. This result suggests that the levels of dissolution of these dopes were different; dope M50 was in a poorly dissolved state, while dope M110 was much closer to a full solution. In other words, although the four dopes had initially a similar macroscopic appearance (that of a clear fluid), those dissolved at lower temperatures might contain more undissolved and invisible pre-nucleation embryos and the higher the density of the latter the earlier a gelation is induced. This is confirmed by X-ray diffraction studies of the dopes for different maturation times. In Fig. 7, XRD diffractograms of the four dopes aged for different times are demonstrated. For dope M50, the diffraction intensity increased with time (Fig. 7a) and the maximum intensity was located over the $2 \theta$ range of $18-20^{\circ}$, consistent with the diffraction peaks of PVDF membrane shown in Fig. 6. In fact, after 4 days of storage, the diffraction pattern
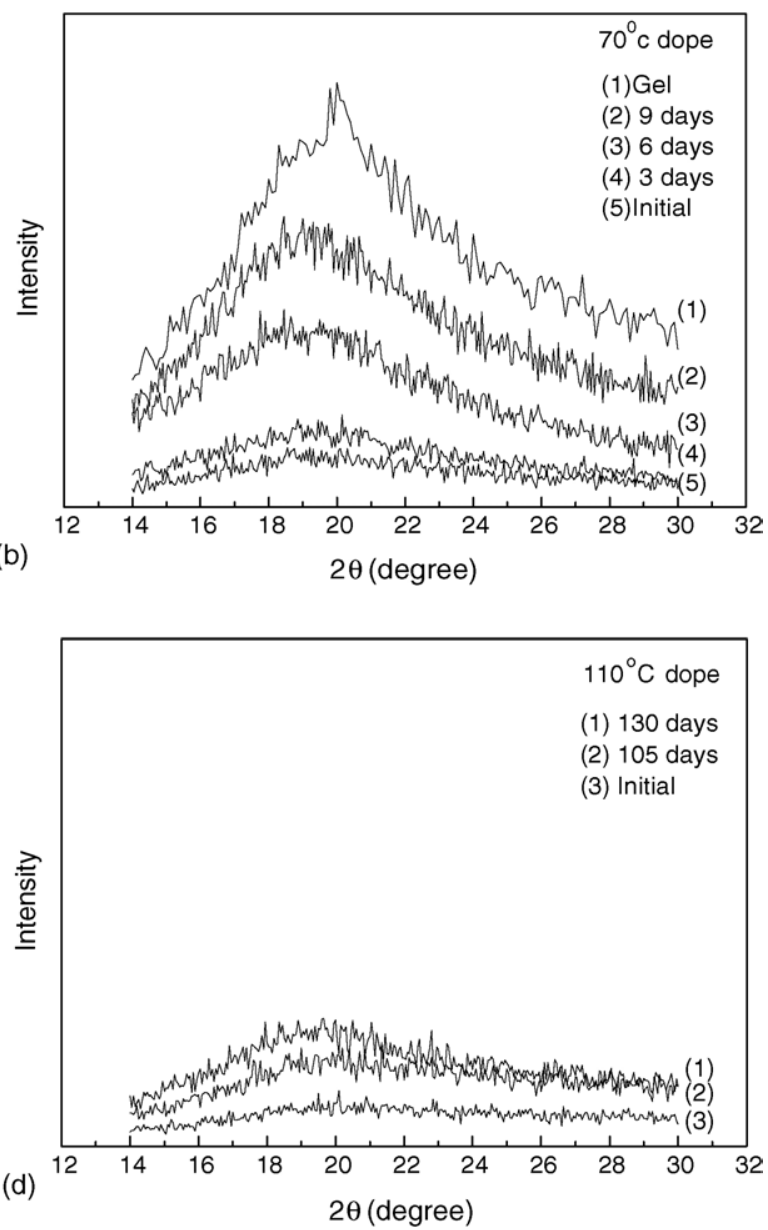

Fig. 7. WAXD patterns of dopes at different aging times. Dopes prepared at (a) $50^{\circ} \mathrm{C}$; (b) $110^{\circ} \mathrm{C}$; (c) $90^{\circ} \mathrm{C} ;\left(\right.$ d) $110^{\circ} \mathrm{C}$. 
was already close to that of the gel, which was formed at the seventh day. Apparently, crystallites have been formed at this stage, as was evident from the small angle light scattering study of similar dopes reported previously [6]. The diffractograms of dopes M70 and M90 (Fig. 7b and c) show a similar trend to that for the M50 dope. However, it takes a much longer time for the diffraction intensity to exhibit a substantial increase, e.g., 60 days for M90 and 9 days for M70. Fig. 7d shows the maturation result for dope M110. It appears that even after 130 days of storage there is only a slight increase of the diffraction intensity, suggesting that the dope is still in a dissolved state close to the original solution. Thus, it can be concluded that the dissolution temperature affects the aggregation state of the dope, which in turn changes the porous structure of the formed membranes.

The occurrence of crystallite-based gelation within the supposedly single-phase region can be explained as follows. A polymer with a distribution of molecular weights is a pseudo-single-component. Hence, true phase boundaries are diffuse and when the system is within but not far from the limits of single-phase boundary, minute amounts of additional phases might be present. In our case, such an additional phase is crystalline (PVDF nano-crystallites) and if a sufficient number of them develop, gelation can take place. Gelation resulting from nano-crystallites as physical-chemical crosslinks is common in the literature of polymer solutionbased gels [25].

An issue of pertinent interest is the influence of dope maturation time on membrane morphology. Fig. 8 presents the SEM images of two typical cases: (a) dope M50 aged for 6 days and (b) dope M90 aged for 80 days. A comparison of Fig. 1a with Fig. 8a indicates that maturation time has a substantial effect in morphology. The globules of Fig. 1a are replaced by a large number of crystallites in the shape of rod or twisted sheet. After being aged for six days, the dope contained a high population of nuclei (cf. XRD in Fig. 7a), which grow and fill-up the space rapidly upon contact with the bath, so that the structure shows only fade traces of supramolecular crystalline aggregates (such as spherulite structures and the like). As a matter of fact, even Fig. 1a globules are quite irregular/imperfect compared to those in Figs. 2 and 3a; hence, Fig. 1a corresponds to a border-case density of nuclei for globular aggregates to form; a further increase of nucleation density upon substantial aging leads to a breaking down of the process of systematic supramolecular aggregation. This is analogous to the case of polymer crystallization from the melt under conditions of substantial undercooling: lamellae do form but no systematic supramolecular aggregates are observed. Fig. 8a shows that the range of structures obtained by controlling the dissolution temperature can be further extended by varying, in addition, the aging time.

Fig. $8 \mathrm{~b}$ can be compared with Fig. 3a. The size of the globules decreased from ca. 3.5-4.0 $\mu \mathrm{m}$ for the non-aged M90 dope to ca. $1.0 \mu \mathrm{m}$, after an 80 days maturation; that is the morphology became intermediate to the M50 and M70 morphologies without long aging. Such a substantial change

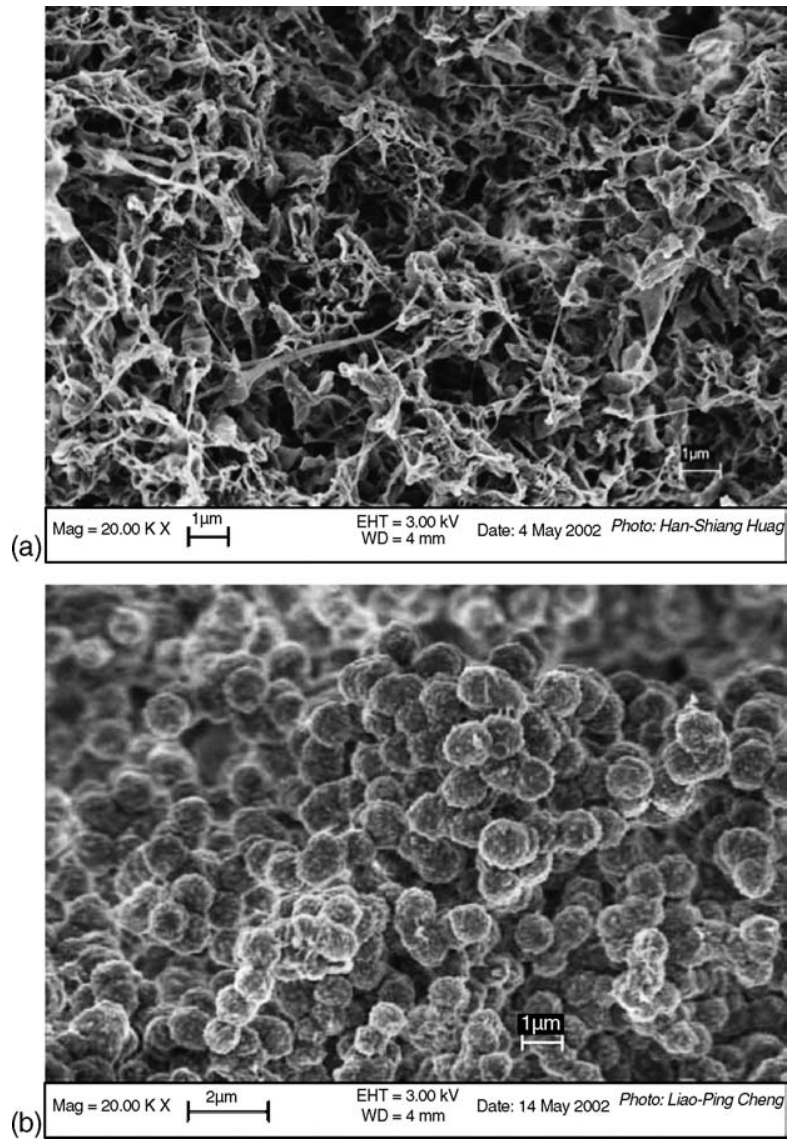

Fig. 8. SEM micrographs of PVDF membranes prepared by the aged dopes. (a) M50 aged for 6 days; (b) M90 aged for 80 days.

in morphology is again attributed to the considerably larger amount of nuclei formed during aging of an incompletely dissolved metastable dope in which pre-nucleation embryos may gradually develop into nuclei during the period of storage. The structure shown in Fig. $8 \mathrm{~b}$ provides a proof of a type of maturation time-dissolution temperature equivalence of ways to control the membrane structure, while the structure of Fig. 8a suggests that such an equivalence can be used to generate membrane structures practically inaccessible through the use of dissolution temperature alone (dissolution times for temperatures substantially below $50{ }^{\circ} \mathrm{C}$ are prohibitively long).

\section{Conclusions}

Microporous PVDF membranes prepared by immersionprecipitation of a DMF/PVDF solution from 1-octanol exhibit interesting semicrystalline morphologies, which typically resemble a packed bed of nearly equal-sized globules. The PVDF crystals present are of $\alpha$-type with a melting temperature of $170{ }^{\circ} \mathrm{C}$ and the membrane material exhibited a heat of fusion of $52 \mathrm{~J} / \mathrm{g}$. The globule size, which reflects the nucleation density of PVDF crystals during the 
course of precipitation, could easily be controlled by an often-overlooked preparation parameter, the dissolution temperature of the casting dope. At a lower dope dissolution temperature, e.g., $50^{\circ} \mathrm{C}$, the globule size in the formed membrane is only $0.2-0.6 \mu \mathrm{m}$, and as the dissolution temperature is raised the size increases considerably; e.g., it becomes as large as $10-50 \mu \mathrm{m}$ when the dope is prepared at $110^{\circ} \mathrm{C}$. Such a dramatic change in nucleation density and globule size is associated with the state of dissolution of the metastable dopes. The maturation study of the dopes by X-ray diffraction method shows that the diffraction intensity increases as the aging time increases until the dopes eventually gel. Also, the dopes dissolved at lower temperatures gel much faster than those dissolved at higher temperatures. For example, it takes ca. 7 days and 82 days to gel, respectively, for dopes prepared at 50 and $90^{\circ} \mathrm{C}$. A study of membranes prepared by sufficiently aged dopes (XRD intensity close to that of the gel) shows that the small globules $(0.5 \mu \mathrm{m})$ in membrane M50 evolve into stick-like crystallites and that the large globules $(3.5-4.0 \mu \mathrm{m})$ in M90 become small ones $(1 \mu \mathrm{m})$. These data demonstrate an equivalence of maturation time and dissolution temperature; hence, a desired porous structure can be produced either by a choice of a proper dissolution temperature alone, or by a more convenient combination of dissolution temperature and maturation time.

\section{Acknowledgement}

The authors would like to thank the National Council of Taiwan for supporting this work through grant NSC91-2216E-032-002.

\section{References}

[1] K. Beltsios, T. Steriotis, K. Stefanopoulos, N. Kanellopoulos, Membrane science and technology, in: F. Schueth, K. Sing, J. Weitkamp (Eds.), Handbook of Porous Solids, Wiley-VCH, 2002, pp. 2281-2433.

[2] M. Mulder, Basic Principles of Membrane Technology, Kluwer Academic Press, Dordrecht, The Netherlands, 1991.

[3] R.E. Kesting, Synthetic Polymeric Membranes, John Wiley and Sons, New York, 1985.

[4] A.M.W. Bulte, M.H.V. Mulder, C.A. Smolders, H. Strathmann, Diffusion induced phase separation with crystallizable nylons. I. Mass transfer processes for nylon 4,6, J. Membr. Sci. 121 (1996) 37.

[5] L.P. Cheng, A.W. Dwan, C.C. Gryte, Membrane formation by isothermal precipitation in polyamide-formic acid-water systems I. Description of membrane morphology, J. Polym. Sci. Polym. Phys. 33 (1995) 211.

[6] L.P. Cheng, T.H. Young, L. Fang, J.J. Gau, Formation of particulate microporous membranes by isothermal immersion-precipitation from the 1-octanol/dimethylformamide/PVDF systems, Polymer 40 (1999) 2395 .
[7] L.P. Cheng, T.H. Young, W.Y. Chuang, L.Y. Chen, L.W. Chen, The formation mechanism of membranes prepared from the nonsolvent-solvent-crystalline polymer systems, Polymer 42 (2001) 443.

[8] D.R. Lloyd, K.E. Kinzer, H.S. Tseng, Microporous membrnae formation via thermally induced phase separation. I. Solid-liquid phase separation, J. Membr. Sci. 52 (1990) 239.

[9] D.R. Lloyd, S.S. Kim, K.E. Kinzer, Microporous membrane formation via thermally-induced phase separation. II. Liquid-liquid phase separation, J. Membr. Sci. 64 (1991) 1.

[10] M. Shang, H. Matsuyama, T. Maki, M. Teramoto, D.R. Lloyd, Preparation and characterization of poly(ethylene-co-vinyl alcohol) membranes via thermally-induced liquid-liquid phase separation, J. Appl. Polym. Sci. 87 (2003) 853.

[11] D.J. Lin, K. Beltsios, C.L. Chang, L.P. Cheng, The fine structure and formation mechanism of particulate phase inversion poly(vinylidene fluoride) membranes, J. Polym. Sci., Part B: Polym. Phys. 41 (2003) 1578.

[12] L.P. Cheng, Effect of temperature on the formation of microporous PVDF membranes by precipitation from 1-octanol/DMF/PVDF and water/DMF/PVDF systems, Macromolecules 32 (1999) 6668.

[13] K. Beltsios, E. Athanasiou, C. Aidinis, N. Kanellopoulos, Microstructure formation phenomena in phase inversion membranes, J. Macromol. Sci. Phys. B38 (1999) 1.

[14] K.G. Beltsios, M.C.M. Bedard, Structure formation phenomena during phase inversion. II. Semiflexible polymers and liquid crystallinity, J. Macromol. Sci. Phys. B39 (2000) 623.

[15] R.C. Ruaan, H.L. Chou, H.A. Tsai, D.M. Wang, J.Y. Lai, Factors affecting the nodule size of asymmetric PMMA membranes, J. Membr. Sci. 190 (2001) 135.

[16] K. Nakagawa, Y. Ishida, Annealing effects in poly(vinylidene fluoride) as revealed by specific volume measurements, differential scanning caloremetry, and electron microscopy, J. Polym. Sci. Polym. Phys. 11 (1973) 2153.

[17] B. Wunderlich, Macromolecular Physics, vol. 1, Academic Press, 1973.

[18] M. Cakmak, A. Teitge, H.G. Zachmann, J.L. White, On-line smallangle and wide-angle $\mathrm{X}$-ray scattering studies on melt-spinning poly(vinylidene fluoride) tape using synchrotron radiation, J. Polym. Sci. Polym. Phys. 31 (1993) 371.

[19] B.-E.E. Mohajir, N. Heymans, Changes in structural and mechanical behaviour of PVDF with processing and thermomechanical treatments 1. Change in structure, Polymer 42 (2001) 5661.

[20] J.B. Lando, H.G. Olf, A. Peterlin, Nuclear magnetic resonance and $\mathrm{X}$-ray determination of the structure of poly(vinylidene fluoride), J. Polym. Sci. A1 4 (1966) 941.

[21] A.J. Lovinger, Poly(vinylidene fluoride), in: D.C. Bassett (Ed.), Developments in Crystalline Polymers-1, Applied Science Publisher, 1988 (Chapter 5).

[22] G.T. Davis, J.E. McKinney, M.G. Broadhurst, S.C. Roth, Electricfield-induced phase changes in poly(vinylidene fluoride), J. Appl. Phys. 49 (1978) 4998.

[23] L.P. Cheng, D.J. Lin, K.C. Yang, Formation of mica-intercalatedNylon 6 nanocomposite membranes by phase inversion method, J. Membr. Sci. 172 (2000) 157.

[24] T.H. Young, D.J. Lin, J.J. Gau, W.Y. Chuang, L.P. Cheng, Morphology of crystalline Nylon-610 membranes prepared by the immersion-precipitation process: competition between crystallization and liquid-liquid phase separation, Polymer 40 (1999) 5011.

[25] J.M. Guenet, Thermoreversible Gelation of Polymers and Biopolymers, Academic Press, London, 1992. 